\author{
International Journal of Innovative Research in \\ Electrical, Electronics, Instrumentation and Control Engineering
}

Vol. 7, Issue 3, March 2019

\title{
Efficient Removal of Noise from Image using Non-Linear Filtering
}

\author{
Tufan Sadhukhan ${ }^{1}$, Sharmistha Chatterjee ${ }^{2}$, Rudra Kanti Das ${ }^{3}$, Surojit Ghosh ${ }^{4}$, \\ Subhadip Roy ${ }^{5}$, Dipshika Das ${ }^{6}$ \\ Student, Department of ECE, Techno International New Town, Kolkata, India ${ }^{1,2,3,4,5}$ \\ Assistant Professor, Department of ECE, Techno International New Town, Kolkata, India ${ }^{6}$
}

\begin{abstract}
An image may contain different types of information related to parameters such as colour and brightness, the irregular variation of which leads to noise in an image. Images often get corrupted by different kind of noise such as Impulse, Gaussian and Salt-and-Pepper Noise, during image acquisition or transmission caused by the digital sensor attempting to record/capture tiny amount of light. Since an image appears in multiple resolutions in different forms, an image can be segmented into different classifying features. As a result, signal and noise can be separated and elimination of noise becomes easier. In this article, a non-linear median filter is preferred, which is found to be useful in reducing not only Impulse Noise but also Gaussian and Speckle Noise. The corresponding analyses are performed on grayscale images, where the results obtained through non-linear filtering are superior to results obtained using Average, Gaussian and Weiner filters.
\end{abstract}

Keywords: Noise, Image, Non-Linear Filter, Gaussian, Salt-And-Pepper, Median Filter

\section{INTRODUCTION}

Removal of noise from an image is known as de-noising. Image de-noising is one of the most important and essential parts of Image Processing [1]. Much scientific data picked up by different sensors and other electronic devices get affected by noise, during image acquisition, due to non-ideal operation of the electronic device or due to any naturally occurring phenomenon. Signal conditioning such as Amplification, A/D Conversion, etc. produce noise due to improper operation of a malfunctioning electronic device. Several types of distortion might exist. Some of the common types that often exist are Gaussian, Impulse and Speckle Noise, where Gaussian Noise is additive, speckle is multiplicative and Impulse Noise is a combination of both [2][3]. At the same time we observe that different kind of noise might exist in a single image, with varying intensities. In the past, many research workers have attempted and succeeded in solving the problem of de-noising. However more and more efforts in this field of research are getting evolved with a view of improving one or more parameters of de-noising.

The current paper deals with the testing of the performance of the non-linear median filtering scheme for image denoising [4]. Section II surveys filters used for removal of noise from images. It then discusses the advantages median filtering has over linear filtering schemes such as Gaussian filtering. Section III describes the proposed median filtering scheme. Section IV compares the simulation results obtained by using median filtering with linear filtering results for the same images. Section V concludes the paper with a discussion on the scope for research in this domain.

\section{LITERATURE SURVEY}

Image de-noising schemes have been researched since the early 1970s, having had the potential for widespread commercial applications [1]. The standard de-noising schemes have mostly relied on application of different types of filters for image reconstruction, augmentation or correction. Initially, linear filters such as Average filters and Gaussian filters have been investigated by researchers [5][6]. It has been observed by researchers that the performance of linear filters depends heavily on the types of noise present in images, such as salt-and-pepper noise and Gaussian noise [7][8]. Linear Filtering is one in which the value of an output pixel is the linear combination of the values of the pixels in the input pixel neighborhood. Moreover it follows Superposition and Linearity Principle. On the other hand, in a Non Linear Filter the value of an output pixel is not a linear combination of the values of the pixel in the input pixel neighborhood. It does not follow Superposition and Linearity principle. Now the question arises that which kind of filter should we use to tackle different kind of noise. In case where the image contains a large amount of noise but the magnitude of it remains low, we prefer a Linear Low pass Filter. Whereas if an image contains a low amount of noise with relatively high magnitude, then any non-linear filter like Median Filter is preferred. 


\section{International Journal of Innovative Research in Electrical, Electronics, Instrumentation and Control Engineering}

Vol. 7, Issue 3, March 2019

In many cases, the solution to de-noising images with high-magnitude low-quantity noise has been achieved through the use of median filter, which is a type of non-linear filter [9].

\section{MEDIAN FILTERING SCHEME}

Median Filtering is a non-linear filtering technique that is well known for its ability to remove different characteristics of noise, while preserving sharp edge. Such noise reduction is a typical pre-processing step to improve the results of later processing (for example, edge detection on an image). Mean Filter replaces the pixel value with the mean of the pixel values but it does not preserve image details. But in Median Filter, the pixel value is not replaced with the mean of the neighborhood pixel values, rather it is replaced with the median of those values. At first the Median is calculated by sorting all the pixel values from the surrounding neighborhood in numerical increasing order and then replacing the pixel being considered with the middle pixel value. Median Filter is the best answer to impulse noise such as Salt and Pepper noise. Salt and Pepper is a form of Impulse Noise, where Salt denotes the Low Frequency noise and Pepper denotes the High Frequency Noise. A median filter finds Salt and Pepper Noise and replaces only the noisy pixels with the median value of the neighborhood around the noise. Similarly after further study it is observed that Median Filter is also the best treatment to Speckle Noise.

Let us consider a $3 \times 3$ image as shown in figure 1 below.

\begin{tabular}{|c|c|c|}
\hline 91 & 55 & 90 \\
\hline 77 & 68 & 95 \\
\hline 115 & 151 & 210 \\
\hline
\end{tabular}

Figure 1: Initial 3x3 Image pixel region

We sort the original image pixel values on the full $3 \times 3$ window getting the sequence 55, 68, 77, 90, 91, 95, 115, 151, 210. Hence 91 is obtained as median value. Now we replace the middle value (68) with the Median value achieved from the previous step, as shown in the following figure 2 .

\begin{tabular}{|c|c|c|}
\hline 91 & 55 & 90 \\
\hline 77 & $\mathbf{9 1}$ & 95 \\
\hline 115 & 151 & 210 \\
\hline
\end{tabular}

Figure 2: Output image pixels after filtering

Similarly we consider the 5x5 image shown below in figure 3.

\begin{tabular}{|l|l|l|l|l|}
\hline 31 & 54 & 89 & 63 & 41 \\
\hline 21 & 36 & 84 & 78 & 61 \\
\hline 22 & 33 & 99 & 74 & 47 \\
\hline 52 & 62 & 14 & 68 & 41 \\
\hline 27 & 39 & 45 & 55 & 81 \\
\hline
\end{tabular}

Figure 3: Initial 5x5 image pixels

Now using the same technique described above, the final image is obtained as shown in figure 4.

\begin{tabular}{|l|l|l|l|l|}
\hline 31 & 54 & 89 & 63 & 41 \\
\hline 21 & $\mathbf{3 6}$ & 84 & 78 & 61 \\
\hline 22 & 33 & $\mathbf{6 8}$ & 74 & 47 \\
\hline 52 & 62 & 14 & 68 & 41 \\
\hline 27 & 39 & 45 & 55 & 81 \\
\hline
\end{tabular}

Figure 4: Final 5x5 image pixels

Median filtering is one kind of smoothing technique, as is linear Gaussian filtering. All smoothing techniques are effective at removing noise in smooth patches or smooth regions of a signal, but adversely affect edges. Often it is important to preserve the edges along with reducing the noise in an image. Edges are of critical importance to the visual appearance of images. For example the edges play a key role in medical and satellite imaging and they must be 


\section{International Journal of Innovative Research in Electrical, Electronics, Instrumentation and Control Engineering}

Vol. 7, Issue 3, March 2019

preserved sharp and undistorted. For small to moderate levels of (Gaussian) noise, the median filter is demonstrably better than Gaussian blur at removing noise whilst preserving edges for a given, fixed window size. This edge preserving property also puts this filter at an advantage over linear filters such as Gaussian and Average filters.

\section{SIMULATION RESULTS}

Now as a Practical Verification of the stated proposition, we put the Reconstructed Image along with the reference image under critical parametric comparison. Here we show that, Median Filter generates the most appropriate output (de-noised image), with Pictorial Experimental Results performed via MATLAB shown below in figure 5:

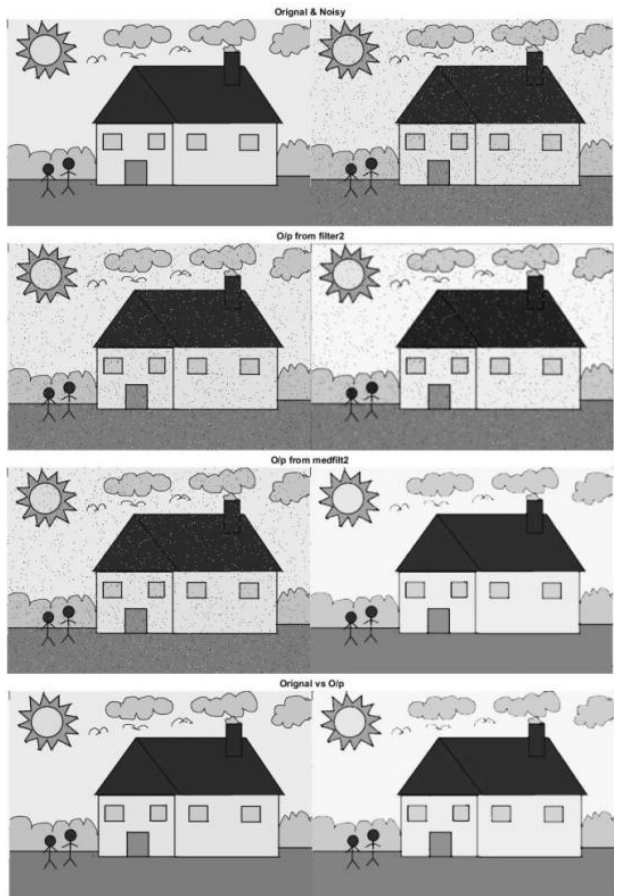

Figure 5: Noising and de-noising of grayscale image

So here we have shown that at first we have put the original fresh image then mixed with noise; after that we have performed basic filtering operation onto that noisy image. It is seen that Median filter gives a clear and the best denoised image. Now the experimental results shown above are supported by practically performed parametric comparison using MATLAB. So we define those parameters as follows:

MSE (Mean Squared Error): One of the error metrics used to compare various image compression techniques is the Mean Square Error (MSE). The MSE is the cumulative squared error between the compressed and the original image. Mathematically it can be represented by-

$$
\text { MSE }=\frac{1}{\mathrm{MN}} \sum_{\mathrm{y}=1}^{\mathrm{M}} \sum_{\mathrm{x}=1}^{\mathrm{N}}\left[\mathrm{I}(\mathrm{x}, \mathrm{y})-\mathrm{I}^{\prime}(\mathrm{x}, \mathrm{y})\right]^{2}
$$

Where $\mathrm{I}(\mathrm{x}, \mathrm{y})$ is the original image, $\mathrm{I}^{\prime}(\mathrm{x}, \mathrm{y})$ is the approximated version (which is actually the decompressed image) and $\mathrm{M}, \mathrm{N}$ are the dimensions of the images. A lower value for MSE means lesser error.

PSNR (Peak Signal to Noise ratio): PSNR can be defined as the parameter used to measure the quality of reconstruction of lossy and lossless compression. It is actually a measure of the peak error. Mathematically it can be represented by-

$$
\begin{aligned}
\text { PSNR } & =10 * \log _{10} \frac{\mathrm{MAXi}^{2}}{\mathrm{MSE}} \\
& =20 * \log _{10} \frac{\mathrm{MAXi}}{\sqrt{\mathrm{MSE}}}
\end{aligned}
$$




\section{International Journal of Innovative Research in Electrical, Electronics, Instrumentation and Control Engineering}

Vol. 7, Issue 3, March 2019

Here, $\mathrm{MAX}_{\mathrm{I}}$ is the maximum possible pixel value of the image. When the pixels are represented using 8 bits per sample, this is 255. More generally, when samples are represented using linear PCM with B bits per sample, MAX $_{\mathrm{I}}$ is $2^{\mathrm{B}}-1$.

Parametric Results: The results of MSE and PSNR values for the final output image are listed in the tables below for comparison with the previous calculated unfiltered values using various noise types at different noise densities.

Table 1: Comparison of MSE values of various filters at different noise densities using salt \& pepper noise

\begin{tabular}{|l|l|l|l|l|l|}
\hline & $\mathbf{1 0 \%}$ & $\mathbf{2 0 \%}$ & $\mathbf{3 0 \%}$ & $\mathbf{4 0 \%}$ & $\mathbf{5 0 \%}$ \\
\hline Before Filtering & 3406.8 & 5289.7 & 6920.3 & 8779.3 & 10589.2 \\
\hline Average Filter & 1387.6 & 1814.3 & 2049.7 & 2449.8 & 2916.8 \\
\hline Gaussian Filter & 1497.9 & 1957.6 & 2682.8 & 2796.5 & 731.0 \\
\hline Wiener Filter & 2851.6 & 2866.4 & 2987.3 & 3012.8 & 339.7 \\
\hline Median Filter & 781.6 & 1021.8 & 1805.9 & 2191.3 & 387.5 \\
\hline
\end{tabular}

Table 2: Comparison of PSNR (in dB) of various filters at different noise densities using salt \& pepper noise

\begin{tabular}{|l|l|l|l|l|l|}
\hline & $\mathbf{1 0 \%}$ & $\mathbf{2 0 \%}$ & $\mathbf{3 0 \%}$ & $\mathbf{4 0 \%}$ & $\mathbf{5 0 \%}$ \\
\hline Before Filtering & 12.65 & 10.47 & 9.68 & 8.45 & 7.89 \\
\hline Average Filter & 15.92 & 14.97 & 15.01 & 14.31 & 13.45 \\
\hline Gaussian Filter & 16.31 & 15.11 & 13.78 & 13.62 & 12.49 \\
\hline Wiener Filter & 13.58 & 13.55 & 12.92 & 13.36 & 12.82 \\
\hline Median Filter & 19.16 & 18.00 & 15.52 & 14.70 & 13.81 \\
\hline
\end{tabular}

We have also performed the same parametric comparison using Gaussian Noise.

Table 3: Comparison of MSE of various filters at different noise densities using Gaussian noise

\begin{tabular}{|l|l|l|l|l|l|}
\hline & $\mathbf{1 0 \%}$ & $\mathbf{2 0 \%}$ & $\mathbf{3 0 \%}$ & $\mathbf{4 0 \%}$ & $\mathbf{5 0 \%}$ \\
\hline Before Filtering & 5291.2 & 6955.9 & 9037.5 & 9507.3 & 10712.6 \\
& & & & & \\
\hline Average Filter & 1814.2 & 2697.1 & 3591.1 & 4133.6 & 4851.6 \\
\hline Gaussian Filter & 1562.7 & 2012.9 & 2494.8 & 2666.7 & 2994.8 \\
\hline Wiener Filter & 3070.3 & 3321.9 & 3550.0 & 3779.6 & 3999.0 \\
\hline Median Filter & 1425.6 & 1910.6 & 2341.9 & 2550.6 & 2760.1 \\
\hline
\end{tabular}

Table 4: Comparison of PSNR (in dB) of various filters at different noise densities using Gaussian noise

\begin{tabular}{|l|l|l|l|l|l|}
\hline & $\mathbf{1 0 \%}$ & $\mathbf{2 0 \%}$ & $\mathbf{3 0 \%}$ & $\mathbf{4 0 \%}$ & $\mathbf{5 0 \%}$ \\
\hline Before Filtering & 10.45 & 9.65 & 8.51 & 8.34 & 7.78 \\
\hline Average Filter & 16.45 & 5.31 & 14.35 & 14.00 & 13.50 \\
\hline Gaussian Filter & 16.10 & 15.10 & 14.23 & 13.54 & 13.12 \\
\hline Wiener Filter & 13.21 & 12.87 & 12.57 & 12.31 & 12.08 \\
\hline Median Filter & 17.87 & 16.98 & 15.80 & 14.25 & 13.58 \\
\hline
\end{tabular}

Hence it can be argued from the results obtained above that median filter is extremely effective in removing impulsetype noise from images, performing much better than corresponding linear filters. 


\section{International Journal of Innovative Research in Electrical, Electronics, Instrumentation and Control Engineering}

Vol. 7, Issue 3, March 2019

\section{CONCLUSION}

The current work accomplished in this paper comprised the comparison of a median filtering technique for de-noising images with other standard linear filtering schemes. The median filter is seen to have better performance compared to the other filters. However, the median filter is not theoretically well-suited to de-noising images with high quantity of noise and hence hybrid schemes involving linear and non-linear filtering in tandem will be investigated in future.

\section{REFERENCES}

[1]. M. Farr, J. Bratton and H. K. Pollehn, "Long life, low noise proximity focused image intensifiers," 1974 International Electron Devices Meeting (IEDM), Washington, DC, 1974, pp. 73-73.

[2]. J. J. Madhura and D. R. R. Babu, "An effective hybrid filter for the removal of Gaussian-impulsive noise in computed tomography images," 2017 International Conference on Advances in Computing, Communications and Informatics (ICACCI), Udupi, 2017, pp. 1815-1820.

[3]. R. I. Sokolov, "Theoretical investigation of Gaussian and non-Gaussian noise masking properties," 20162 nd International Conference on Industrial Engineering, Applications and Manufacturing (ICIEAM), Chelyabinsk, 2016, pp. 1-4

[4]. C. Chang, J. Hsiao and C. Hsieh, "An Adaptive Median Filter for Image Denoising," 2008 Second International Symposium on Intelligent Information Technology Application, Shanghai, 2008, pp. 346-350.

[5]. X. Qinlan and C. Hong, "Image Denoising Based on Adaptive Filtering and Multi-frame Averaging Filtering," 2009 International Conference on Artificial Intelligence and Computational Intelligence, Shanghai, 2009, pp. 523-526.

[6]. A. Jain and R. Gupta, "Gaussian filter threshold modulation for filtering flat and texture area of an image," 2015 International Conference on Advances in Computer Engineering and Applications, Ghaziabad, 2015, pp. 760-763.

[7]. L. Hou, H. Liu, Z. Luo, Y. Zhou and T. Truong, "Image deblurring in the presence of salt-and-pepper noise," 2017 IEEE International Conference on Image Processing (ICIP), Beijing, 2017, pp. 2389-2393.

[8]. J. J. Madhura and D. R. R. Babu, "An effective hybrid filter for the removal of Gaussian-impulsive noise in computed tomography images," 2017 International Conference on Advances in Computing, Communications and Informatics (ICACCI), Udupi, 2017, pp. 1815-1820.

[9]. Xiao Kang, Wei Zhu, KeJie Li and Jing Jiang, "A Novel Adaptive Switching Median filter for laser image based on local salt and pepper noise density," 2011 IEEE Power Engineering and Automation Conference, Wuhan, 2011, pp. 38-41. 